\title{
DEVELOPMENT OF THE INNATE IMMUNE RESPONSE AND INFLUENCE OF COLOSTRUM SUCKLING IN CALVES
}

\author{
${ }^{1}$ Viviani Gomes, ${ }^{1}$ Camila Costa Baccili, ${ }^{1}$ Vinicius Alvim Passos Baldacim, \\ ${ }^{2}$ Karina Medici Madureira, ${ }^{3}$ Aline Gil Alves Guilloux, \\ ${ }^{4}$ Claudia Rodriguez Pozzi and ${ }^{5}$ Cristina de Oliveira Massoco Salles Gomes \\ ${ }^{1}$ Departament of Clinical Sciences, \\ Faculty of Veterinary Medicine and Animal Science, University of Sao Paulo, Sao Paulo, Brazil \\ ${ }^{2}$ Department of Anatomy, Patology and Veterinary Clinics, \\ School of Veterinary Medicine and Animal Science, Federal University of Bahia, Salvador, Brazil \\ ${ }^{3}$ Departament of Preventive Veterinary Medicine and Animal Healthy, \\ Faculty of Veterinary Medicine and Animal Science, University of Sao Paulo, Sao Paulo, Brazil \\ ${ }^{4}$ Institute of Animal Science, Sao Paulo Agency of Agribusiness Technology, Nova Odessa, Brazil \\ ${ }^{5}$ Departament of Pathology, \\ Faculty of Veterinary Medicine and Animal Science, University of Sao Paulo, Sao Paulo, Brazil
}

Received 2014-03-07; Revised 2014-04-11; Accepted 2014-04-14

\begin{abstract}
Bovine colostrum contains immune factors, like leukocytes and cytokines that are absorbed by the intestinal mucosa of the neonate, however, not much is known about the function of some of these components in the active immune response of calves. The aim of this study was to determine the influence of colostrum intake and development of the innate immune response of calves from birth to two months of age, by evaluating the proportion of neutrophils $\mathrm{CH}_{138^{+}}$and monocytes $\mathrm{CD}_{1}{ }^{+}$, using flow citometry and measurement of proinflammatory cytokines IL-1 beta, IL-6, TNF-alpha and IFN-gamma, using comercial ELISA kits. According to the obtained results we can determine that colostrum intake had influenced the IFN-gamma concentration and the monocytes $\mathrm{CD}_{14}{ }^{+}$and neutrophils $\mathrm{CH} 138^{+}$proportions in the circulation of calves and that these components seem to play a key role in the development of immune response of these animals, providing protection against pathogens, enhancing the phagocytic activity and antigen presentation by monocytes.
\end{abstract}

Keywords: Cytokines, Monocytes, Neutrophils, Flow Cytometer, Bovine

\section{INTRODUCTION}

The innate immune system of fetus and newborn calves is extremely important as it is independent of prior antigenic exposure and immune maturation. Thus, this system represents the main defense mechanism, considering that the development of specific immunity requires time for maturation of lymphocytes after successive exposures to pathogens (Barrington and Parish, 2001; Chase et al., 2008).

High levels of serum estrogen and maternal-fetal cortisol produced at the end of pregnancy and calving have immunosuppressive effects on the cellular components that participate in the innate Immune Response (IR). At this time, despite a transient increase in number of polymorphonuclear leukocytes (PMN), there is a decrease of their functional activity, considering the rates of phagocytosis and bactericidal capacity (Benesi et al., 2012; Chase et al., 2008; Morein et al., 2002).

In this period of immune maturation, maternal colostrum promotes temporary protection to newborn, due to passive transfer of immune factors such as immunoglobulins, leukocytes, hormones and cytokines (Silva et al., 2013), reducing the risk of outbreak of Corresponding Author: Viviani Gomes, Department of Clinical Science, Faculty of Veterinary Medicine and Animal Science, University of São Paulo, São Paulo, Brazil Tel: 55-11-30911331 Email: viviani.gomes@usp.br 
diseases, such as diarrhea (Razzaque et al., 2009). However, the biological function of some of these components and their interrelationship with the active immunity of calves is still poorly known.

Innate immune system cells correspond to $82.8 \%$ of the cellular components of first milking colostrum, $13.3 \%$ of these are Neutrophils (NE) and $69.5 \%$ are Macrophages (MO)/epithelial cells (Gomes et al., 2011). Review about neonatal immunology from Barrington and Parrish (2001) hypothesized that PMN leukocytes are important for cytoplasmic transport of immunoglobulins to the bloodstream of newborn calves, protecting them from enzymatic digestion during its passage through the gastrointestinal tract.

The high proportion of macrophages in colostrum of first milking has intrigued researchers and recent research has shown that these cells may be the key to the activation of specific immune system of calves, by the production of cytokines and presentation of antigens to immature lymphocytes located in secondary lymphoid organs (Reber et al., 2008).

Proinflammatory cytokines IL-1 beta, IL-6, TNF-alpha and IFN-gamma are also present in maternal colostrum, believing in the possibility of its absorption by the intestinal mucosa of calves like the immunoglobulins form, reaching highest concentrations in newborns bloodstream in the first $72 \mathrm{~h}$ of life (Madureira, 2011). The role of these substances in calves immune response is still not fully clear (Chase et al., 2008).

Despite the importance of the components of innate immune response on the protection of calves during their immune maturation, few longitudinal studies have been done to evaluate the influence of colostrum intake and oscilations in the valuation parameters of nonspecific immunity. Therefore, the aim of this study was to determine the influence of colostrum intake and development of the innate immune response of calves from birth to two months of age by evaluating the proportion of neutrophils $\mathrm{CH} 138^{+}$and monocytes $\mathrm{CD} 14^{+}$ and measurement of proinflammatory cytokines.

\section{MATERIALS AND METHODS}

Eleven healthy calves were followed from birth to two months of age, who received fresh colostrum from their mothers, for a total amount of four liters, assigned into two feedings administered within the first $12 \mathrm{~h}$ after birth (a.b). The calves were kept in collective calf pens until weaning at 60 days of life, where they received fresh milk in amount equivalent to $10 \%$ of body weight/day, concentrate, hay and water ad libitum. The general clinical examination of animals was carried out in moments of provided ratings according to the procedures described by Dirksen et al. (1993).

Blood samples from newborn calves were collected into tubes with the $10 \%$ anticoagulant Ethylene Diamine Tetraacetic Acid (EDTA) to determine the proportions of neutrophils $\mathrm{CH} 138^{+}$and monocytes $\mathrm{CD} 14^{+}$and in tubes without anticoagulant for measurement of proinflammatory cytokines. Evaluations were performed at the following times: Before colostrum intake (T0), 3-4 days (T1), 5-6 days (T2), 7-8 days (T3), 9-10 days (T4), 15 days (T5), 30 days (T6) and 60 days after birth (T7).

The proportions of neutrophils $\mathrm{CH}_{138^{+}}$and monocytes $\mathrm{CD}_{14}{ }^{+}$was determined by flow cytometry. The volume relative to $100 \mu \mathrm{L}$ of whole blood was lysed using the comercial solution FACSLyse ${ }^{\circledR}$ (Becton Dickinson Biosciences, San Jose, CA, USA). To leukocytes labeling it was added the primary monoclonal antibodies anti-bovine CH138 (CH138A) and CD14 (MM61A) (VMRD, Pullman, WA), incubated for $30 \mathrm{~min}$ at $4^{\circ} \mathrm{C}$. After that, the cells were washed and labeled with secondary monoclonal antibody anti-mouse, using the FITC (Fluorescent Isothiocyanate) and PECY-5 (Phycoerythrin-Cy5) fluorochromes (Invitrogen Corporation, USA). The samples were incubated one more time for $30 \mathrm{~min}$ in the dark at $4{ }^{\circ} \mathrm{C}$ and again washed with phosphate buffered saline.

Fifty thousand cells per sample were analyzed in FACSCalibur flow cytometer (Becton Dickinson Biosciences, San Jose, CA, USA) using the CellQuest Pro ${ }^{\circledR}$ software (Becton Dickinson Biosciences, San Jose, CA, USA). The evaluation of the fluorescence emitted by the secondary monoclonal antibodies were made using the FlowJo ${ }^{\circledR}$ program (Tree Star, Ashland, OR, USA), version 7.2.5 as described in Fig. 1.

The blood samples of calves were collected in tubes without anticoagulant and were centrifuged at 700xg at $4^{\circ} \mathrm{C}$ to obtain the serum, placed in cryovials and stored at $80^{\circ} \mathrm{C}$ until processing. This material was used for the measurement of Interleukin 1-beta (IL-1 beta), interleukin 6 (IL-6), Tumour Necrosis Factor alpha (TNF-alpha) and Interferon gamma (IFN-gamma) proinflammatory cytokines through enzyme immunoassays using commercial kits for the human species (Human IL-1 beta ELISA Set II, human IL-6 ELISA Set, human TNF-alpha ELISA SEt, human IFN-gamma ELISA Set, BD Biosciences ${ }^{\circledR}$ - San Diego, CA, USA).

The proinflammatory cytokines IL1-beta, IL-6 and TNF-alpha showed no detectable levels in some of the evaluated times, opting for the descriptive analysis of their frequencies and presentation of the maximum values reached

$(\mathrm{pg} / \mathrm{mL})$. 
Viviani Gomes et al. / American Journal of Animal and Veterinary Sciences 9 (2): 77-83, 2014

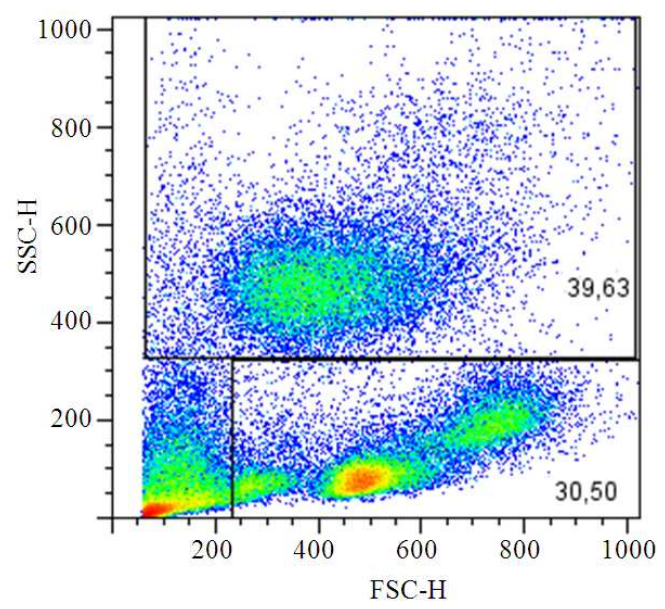

(A)

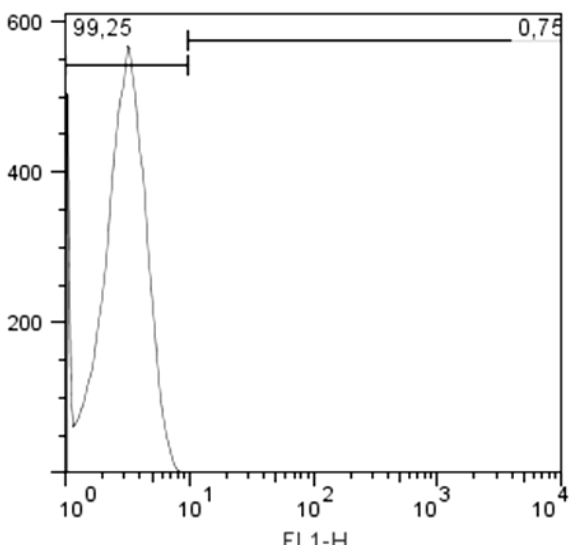

(B)

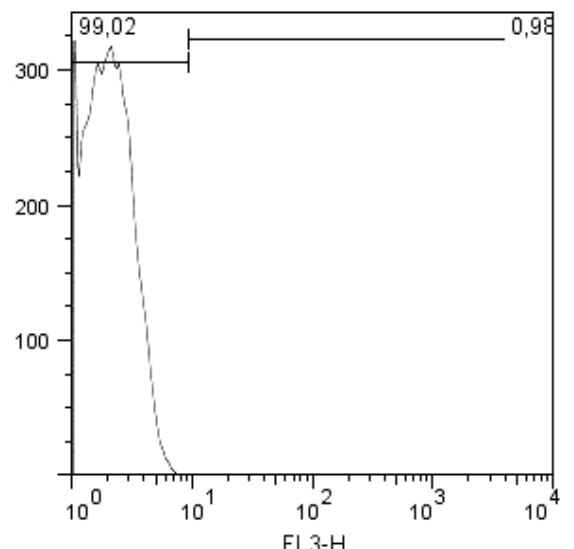

(D)

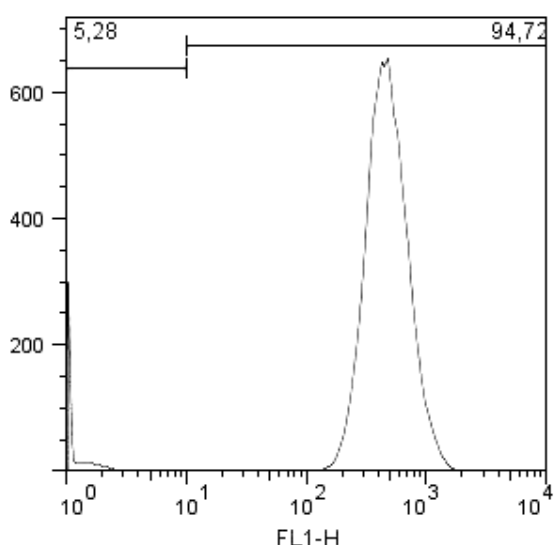

(C)

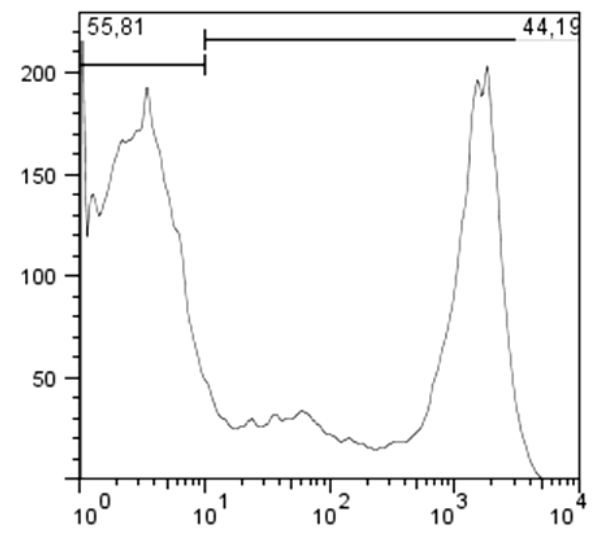

(E)

Fig. 1. The gate was added according to the size (FSC) and granularity (SSC) of the cells (A). Polymorphonuclear Position: 200-1000 FSC-H/0-350-1000 SSC-H (A); Mononuclear Position: 250-1000 FSC-H/0-350 SSC-H (A), the limit between negative and positive population for the marker CH138+ (FL1) are shown in histograms (B) and (C) and for the marker CD14+ (FL3) in the histograms (D) and (E) 
Statistical analysis was performed using the SPSS 20.0 (IBM Corporation, Armonk, NY). The variables were tested by the Kolmogorov-Smirnov test and some had non-normal distribution, opting for non-parametric tests. Differences between the evaluation moments were obtained by the Friedman test and multiple comparisons by Wilcoxon test. All tests were considered significant when $\mathrm{p}<0.05$.

\section{RESULTS}

The absolute and relative values of the positive results for the proinflammatory cytokines are expressed in Table 1. The most of the evaluated animals showed negative results, especially for IL-1beta and IL-6 cytokines. TNF-alpha and IFN-gamma were the most detected cytokines in bloodstream of calves from the time $\mathrm{T} 1$.

The median values of the ratios (\%) of neutrophils $\mathrm{CH} 138^{+}$and monocytes $\mathrm{CD} 14^{+}$in blood of calves from birth to two months of age are shown in Fig. 2.
Variations in the concentrations of TNF-alpha $(\mathrm{P}=$ 0.035), IFN-gamma $(\mathrm{P}=0.002)$, neutrophils $(\mathrm{P}=$ $0.001)$ and monocytes $(\mathrm{P}=0.004)$ were observed according to the Friedman test. The IL-1beta $(\mathrm{P}=$ $0.261)$ and IL-6 $(\mathrm{P}=0.271)$ cytokines showed no fluctuations during the studied period.

The comparison between the moments $\mathrm{T} 0$ and $\mathrm{T} 1$ showed a significant difference $(P=0.028)$ only for the IFN-gamma. No significance between T0 and T1 for IL1 beta $(\mathrm{P}=0.068)$, IL-6 $(\mathrm{P}=0.317)$, TNF-alpha $(\mathrm{P}=$ $0.109)$, neutrophils $\mathrm{CH} 138^{+}(\mathrm{P}=0.953)$ and monocytes CD14 ${ }^{+}$cells $(\mathrm{P}=0.260)$ was observed.

The comparison between the times for IFN-gamma revealed a lower value obtained at $\mathrm{T} 0$ when compared with other periods evaluated in this study $(\mathrm{p}<0.05)$, except for T5. The IFN-gamma concentration obtained in T5 was also lower than T2 to T4. Thus, it was observed an IFN-gamma increase after colostrum intake with stability values until $\mathrm{T} 4$, decrease at T5 and later increase at T7. Regarding the cytokine TNF-alpha, a difference was observed only in T3, which was higher than T0, T5 and T6.
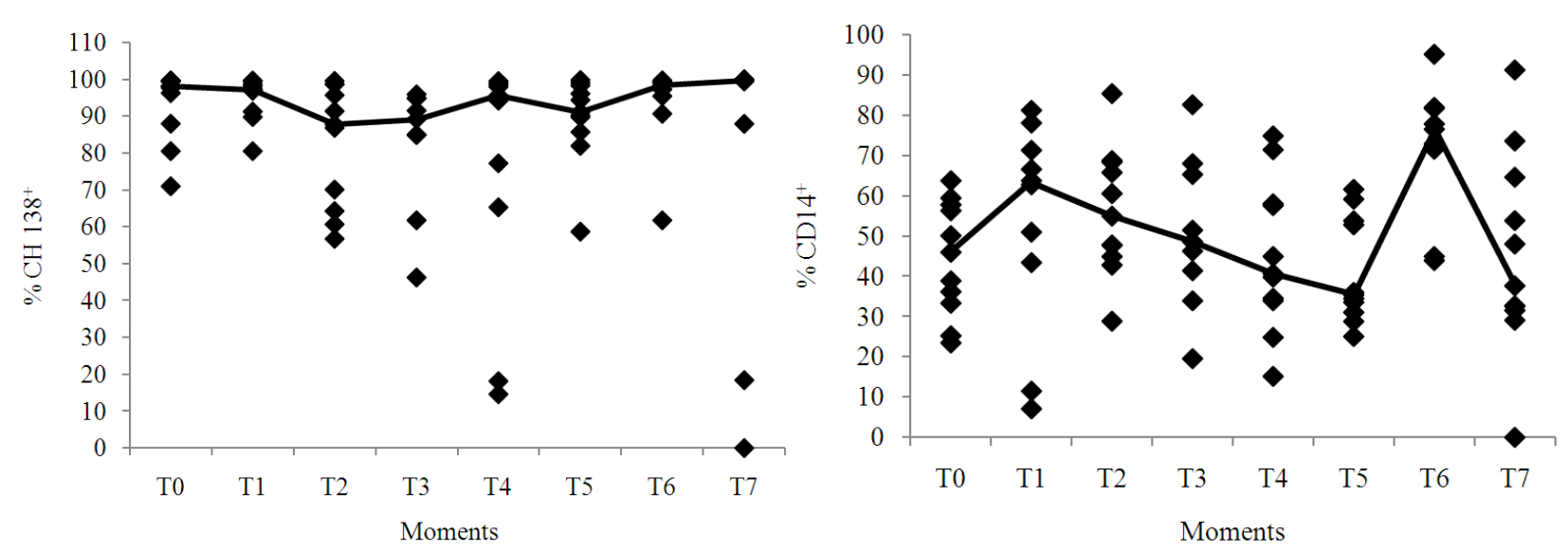

Fig. 2. Median and individual values of the relative percentages of neutrophils $\mathrm{CH} 138^{+}$(A) and monocytes $\mathrm{CD} 14^{+}(\mathrm{B})$ in the blood of calves from birth to two months of life

Table 1. Absolute Value (AV), rate (\%) of positive samples, maximum values ( $\mathrm{pg} / \mathrm{mL})$ and median $(\mathrm{pg} / \mathrm{mL})$ for proinflammatory cytokines in the blood of calves from birth (T0) at two months of life (T7)

\begin{tabular}{|c|c|c|c|c|c|c|c|c|c|c|c|c|c|}
\hline \multirow[b]{2}{*}{ Moments } & \multirow[b]{2}{*}{ AV } & \multicolumn{2}{|c|}{ IL-1 beta } & \multirow[b]{2}{*}{ AV } & \multicolumn{2}{|r|}{ IL-6 } & \multirow[b]{2}{*}{ AV } & \multicolumn{2}{|r|}{ TNF-alpha } & \multirow[b]{2}{*}{ AV } & \multicolumn{2}{|r|}{ IFN-gamma } & \multirow[b]{2}{*}{ Median } \\
\hline & & $\%$ & Maximum value & & $\%$ & Maximum value & & $\%$ & Maximum value & & $\%$ & Maximum value & \\
\hline T0 & 0 & 0 & 0 & 0 & 0 & 0 & 0 & 0 & 0 & 1 & 9,09 & 7,5 & 0 \\
\hline $\mathrm{T} 1$ & 4 & 36,36 & 12,3 & 0 & 0 & 0 & 3 & 27,27 & 23,4 & 6 & 54,54 & 33,3 & 0,7 \\
\hline $\mathrm{T} 2$ & 1 & 9,09 & 3,5 & 1 & 9,09 & 0,5 & 3 & 27,27 & 24,9 & 9 & 81,82 & 18,7 & 10 \\
\hline $\mathrm{T} 3$ & 1 & 9,09 & 5,4 & 2 & 18,18 & 60,9 & 5 & 45,45 & 37,8 & 6 & 54,54 & 62,3 & 3,3 \\
\hline $\mathrm{T} 4$ & 2 & 18,18 & 3,2 & 2 & 18,18 & 60,0 & 3 & 27,27 & 6,8 & 7 & 63,64 & 46,4 & 11,7 \\
\hline $\mathrm{T} 5$ & 0 & 0 & 0 & 0 & 0 & 0 & 1 & 9,09 & 0,3 & 2 & 18,18 & 3,2 & 0 \\
\hline T6 & 2 & 18,18 & 6,5 & 0 & 0 & 0 & 0 & 0 & 0 & 5 & 45,45 & 45,3 & 0 \\
\hline $\mathrm{T} 7$ & 0 & 0 & 0 & 0 & 0 & 0 & 1 & 9,09 & 2,5 & 7 & 63,64 & 26,6 & 8,4 \\
\hline
\end{tabular}


The neutrophils $\mathrm{CH} 138^{+}$proportion was different between T0 Vs T1 $(\mathrm{P}=0.026)$ and T2 $(\mathrm{P}=0.021)$ it was still observed a decrease in the values between $\mathrm{T} 1$ and $\mathrm{T} 2(\mathrm{P}=0.028)$, then the values remained constant until T5 (15 days). The ratios obtained in T6 were higher than $\mathrm{T} 2(\mathrm{P}=0.028)$ and $\mathrm{T} 3(0.018)$ (Fig. 2A).

The monocytes $\mathrm{CD} 14^{+}$showed an increase in $\mathrm{T} 0$ to $\mathrm{T} 1$, despite the lack of statistical difference, which was higher than T2 $(\mathrm{P}=0.008), \mathrm{T} 3(\mathrm{P}=0.041)$ and T4 $(\mathrm{P}=0.06)$, then the values increased again yielding peak at time $\mathrm{T} 6$, which were higher than those obtained in $\mathrm{T} 2(\mathrm{P}=0.028), \mathrm{T} 3$ (0.008) and T4 (0.008). The moment T7 was similar to T5 value but the value was higher than those obtained at T0 $(\mathrm{P}$ $=0.016), \mathrm{T} 1(\mathrm{P}=0.007), \mathrm{T} 2(\mathrm{P}=0.021), \mathrm{T} 3(\mathrm{P}=0.003), \mathrm{T} 4$ $(\mathrm{P}=0.003)$, T6 ( $\mathrm{P}=0.041)$ (Fig. 2B).

\section{DISCUSSION}

The transfer of the newborn calf from the womb to the environment is a challenge to his immune system because sindesmocorial bovine placenta is impermeable to the passage of maternal-fetal macromolecules, which implies hypogammaglobulinemia and immunological immaturity at birth. Thus, the maternal protection during pregnancy postpones the full establishment of specific immunity, since lymphocyte maturation requires successive contacts with pathogens (Barrington and Parish, 2001; Chase et al., 2008; Silva et al., 2013). In this scene, the innate immune system is extremely important for fetus and newborn calves because of their antigenic independence, however, hormones released during late pregnancy and at calving are immunosuppressive and negatively influence this response (Chase et al., 2008; Morein et al., 2002). Remains therefore the colostrum intake for the immune protection supply that will ensure the survival of calves in their first months of life (Kelly, 2003; Razzaque et al., 2009).

In this study, we could observe the influence of colostrum intake in the concentration of IFN-gamma. Despite the lack of statistical difference, it can be seen that the calves were negative for all proinflammatory cytokines before the ingestion of colostrum, but some animals reacted positively to TNF-alpha and IL-1beta after colostrum administration. The absorption mechanism of these colostral cytokines by the intestinal mucosa of the neonate should behave similarly to what happened with the immunoglobulins through the decrease of cells able to expressing the receptors for these proteins with the advancing of post-birth time (Barrington and Parish, 2001).

At time T0 the major of proinflammatory cytokines was undetectable in addition; the lower value for IFN- gamma and TNF-alpha was obtained in T0 in relation to other moments which confirms no inflamation until the parturition time. In contrast, a calf had IFN-gamma levels prior to colostrum intake, probably by antigen contact during pregnancy and despite the uterine environment being sterile, some microorganisms can cross the placental barrier and the innate immune system in these calves is the main defense mechanism during the pregnancy period. Furthermore, the production of IFN-gamma occurs after three days of exposure to pathogens (Barrington and Parish, 2001; Chase et al., 2008).

The transfer of IFN-gamma to calves by colostrum intake may be crucial for its cell-mediated response and protection against intracellular pathogens, since this response is deficient at birth. This substance is secreted primarily by $\mathrm{T}$ cells and Natural Killer (NK) cells, mainly by $\mathrm{CD}^{+}$cytotoxic $\mathrm{T}$ lymphocytes responsible for production of quantities four times higher than the produced by $\mathrm{T}$ helper $\mathrm{CD}^{+}$cells. Interestingly, the predominant $\mathrm{T}$ cells in colostrum at birth are cytotoxic lymphocytes, however, the IFN-gamma cytokine decreases with the progression of lactation, being undetectable on the seventh day postpartum (Chase et al., 2008; Hagiwara et al., 2001).

Although no statistical difference was observed, it can be seen an increase in the monocytes CD14 proportion after colostrum intake. Macrophages are the predominant cells in bovine colostrum and have potential for absorption by the intestinal mucosa of newborn calves when this food is offered as the fresh way in the first $12 \mathrm{~h}$ postpartum (Gomes et al., 2011; Liebler-Tenorio et al., 2002). Besides enhancing the phagocytic response of calves, the colostral macrophages may be the key to the activation and maturation of their specific immune response by increasing the capacity of monocytes to present antigens (Reber et al., 2008).

The association between the obtained results for $\mathrm{CD} 14^{+}$ cells and IFN-gamma allows us to declare that these factors may act in synergy in the development of immune newborn response, since IFN-gamma is the main macrophage activating cytokine, accentuating its microbicide function, by the activation of genes transcription that encoding enzymes phagocyte oxidase and synthase, inductor of the production of nitric oxide, increasing the bactericidal and phagocytic cell activity, which appears reduced in calves as a result of stress occurred at birth (Barrington and Parish, 2001). Studies have shown that monocytes of calves evaluated within one hour after birth exhibited greater bacterial uptake when compared to monocytes from calves with three to nine weeks of life, emphasizing the possible synergism between IFN-gamma and CD14 ${ }^{+}$ cells (Menge et al., 1998). 
The proportions of neutrophils $\mathrm{CH} 138^{+}$were higher on the first day of life and remained high until the sixth day compared to subsequent moments until T5 (15 days). Most studies involving polymorphonuclear leukocytes in calves used leucogram despite the different techniques employed, these studies also found higher numbers of neutrophils in the first days of life (Benesi et al., 2012; Menge et al., 1998). Changes in the population of $\mathrm{CH} 138^{+}$cells in the first days of life may be associated with the stress at delivery and birth, especially because some animals in this study were pulled at birth, increasing the blood concentration of endogenous steroids and migration of these cells from the marginal pool to circulation (Benesi et al., 2012). Therefore, the major proportion of circulating neutrophils recorded before the colostrum intake and in the first days of life of the calves may be a mechanism of immune hand, since many functions related to these cells are immature or incomplete (Menge et al., 1998).

A higher proportion of neutrophils $\mathrm{CH}_{138^{+}}$in the first days of life are not associated with inflammatory processes, because the levels of proinflammatory cytokines were low or undetectable in most calves of this research. The cytokine IL-1beta is a potent mediator of inflammation and acts in synergism with IL-6 and TNF-alpha (Richards, 1998). It is possible that some mothers had mastitis, a common disease in dairy cows in the peripartum period and transferred the proinflammatory cytokines to their calves (Souza et al., 2012). In this case, these cytokines have no influence on the immunity of calves, since most of the calves in this study had undetectable reactions at 15 days (T5) of life, similar indeed to that found by Goto et al. (1997) and Yamanaka et al. (2003) and could direct us to determine the duration of the maternal immune component in the bloodstream of calves.

The secretion of cytokines is characterized by being a short event and is not being stored as a pre-formed molecules and their synthesis is initiated by new gene transcripts as a result of cellular activation. Then, cytokines are rapidly secreted, with IL- 6 having a half life of 5 to 6 days, IFN-gamma from 2 to 4 days and TNF-alpha of 20 min (Nguyen et al., 2007), which could justify the low permanence of these cytokines in the circulation of neonates of this study.

At 30 days of life (T6) inflammatory response was observed for the largest proportion of neutrophils $\mathrm{CH}_{138^{+}}$and monocytes $\mathrm{CD}_{1}{ }^{+}$, associated with the detection of IL-1 beta and IFN-gamma in the serum of some calves. At this stage of the research, some calves developed diarrhea episodes, so it is believed that in this time ocurred an active response by natural exposure to pathogens, because the calves were submitted to a management system of dairy farm and kept in collective calf pens. The phase and intensity of inflammation in agreement with the time of harvest may have influenced the lack of detectable levels of IL-6 and TNF-alpha at this time (T6). However, few studies that measured proinflammatory cytokines in the calves serum assessed animals over a period of fifteen (Goto et al., 1997) and 28 days (Yamanaka et al., 2003).

Peak concentrations obtained for the cytokines were lower than the values-obtained by Goto et al. (1997), who measured the cytokine IL-1 beta in serum of healthy Holstein calves, finding average values of $1.5 \mathrm{ng} \mathrm{mL}^{-1}$ and Yamanaka et al. (2003) who evaluated the IL-1beta, IL-6, TNF-alpha, IFN-gamma cytokines, obtaining mean valuesof $46.9 \pm 31.4,1.23 \pm 0.34,0.69 \pm 0.22$ and $0.192 \pm 0.04 \mathrm{ng}$ $\mathrm{mL}^{-1}$, respectively. Both authors mentioned used bovine ELISA kits in their determinations. In this study, the median values of cytokines were obtained through the use of human ELISA kits because of difficulties in obtaining kits for bovine. Thus, it was considered the homology between the amino acid sequence of human and bovine cytokines (Scheerlinck, 1999), but even there homologies between human cytokines, we found differences between the results obtained by the authors cited above. Madureira (2011), measuring the same proinflammatory cytokines in human kits obtained values close to those obtained in the present work.

The fact of this longitudinal study has been designed in the field resulted in a significant antigenic diversity, as manifested by the observed fluctuations from birth to weaning. Thus, studies on the evaluation of specific immune responses to pathogens that causes diseases in the neonatal period, through experimental infection need to be conducted.

\section{CONCLUSION}

The maternal colostrum intake influence blood concentrations of IFN-gamma, monocyte $\mathrm{CD} 14^{+}$and neutrophils $\mathrm{CH} 138^{+}$in calves evaluated until the first two months of life and that these components may be responsible for the enhancement of the cellular immune response and protection against pathogens, which are deficient at birth, beyond the specific immunity, by increasing the capacity of monocytes to present antigens.

\section{ACKNOWLEDGEMENT}

The researchers are grateful to Fundação de Amparo à Pesquisa do Estado de São Paulo for financial support. 


\section{REFERENCES}

Barrington, G.M. and S.M. Parish, 2001. Bovine neonatal immunology. Vet. Clin. North Am. Food Animal Pract., 17: 463-476. PMID: 11692503

Benesi, F.J., C.M.C. Teixeira, M.L.R. Leal, J.A.N. Lisboa and R.M.S. Mirandola et al., 2012. Leukograms of healthy Holstein calves within the first month of life. Pesquisa Veterinária Brasileira, 32: $\quad 352-356 . \quad$ DOI: $10.1590 /$ S0100736X2012000400013

Chase, C.C.L., D.J. Hurley and A.J. Reber, 2008. Neonatal immune development in the calf and its impact on vaccine response. Vet. Clin. North Am. Food Animal Pract., 24: 87-104. DOI: 10.1016/j.cvfa.2007.11.001

Dirksen, G., H.D. Gründer and M. Stöber, 1993. Exame Clínico dos Bovinos. 3rd Edn., Guanabara Koogan, Rio de Janeiro, ISBN: 852770255X, pp: 419.

Gomes, V., K.M. Madureira, A.M.M.P. Della Libera and M.G. Blagitz et al., 2011. Cell dynamics of Holstein cow colostrum immediately after parturition. Arquivo Brasileiro de Medicina Veterinária e Zootecnia, 63: 1047-1053. DOI: 10.1590/S010209352011000500001

Goto, M., M. Maruyama, K. Kitadate, R. Kirisawa and Y. Obata et al., 1997. Detection of interleukin-1 beta in sera and colostrum of dairy cattle and in sera of neonates. J. Vet. Med. Sci., 59: 437-441. PMID: 9234217

Hagiwara, K., H. Yamanaka, H. Higuchi, H. Nagahata, R. Kirisawa and H. Iwai, 2001. Oral administration of IL-1 $\beta$ enhanced the proliferation of lymphocytes and the $\mathrm{O} 2$ production of neutrophil in newborn calf. Vet. Immunol. Immunopathol., 81: 59-69. PMID: 11498247

Kelly, G.S., 2003. Bovine colostrums: A review of clinical uses. Alternative Med. Rev., 8: 378-394. PMID: 14653766

Liebler-Tenorio, E.M., G. Riedel-Caspari and J.F. Pohlenz, 2002. Uptake of colostral leukocytes in the intestinal tract of newborn calves. Vet. Immunol. Immunopathol., 85: 33-40. DOI: 10.1016/S01652427(01)00404-4

Madureira, K.M., 2011. Concentration of cytokines IL$1 \beta$, IL-6, TNF- $\alpha$ and IFN- $\gamma$ in blood and colostrum of Holstein cows. Importance in the immune passive transfer. PhD Thesis, Universidade de São Paulo, São Paulo, Brazil.
Menge, C.H., B. Neufeld, W. Hirt, N. Schemeer and R. Bauerfeind et al., 1998. Compensation of preliminary blood phagocyte immaturity in the newborn calf. Vet. Immunol. Immunopathol., 62: 309-321. DOI: 10.1016/S0165-2427(98)00109-3

Morein, B., I. Abusugra and G. Blomqvist, 2002. Immunity in neonates. Vet. Immunol. Immunopathol., 87: 207-213. DOI: 10.1016/S01652427(02)00078-8

Nguyen, T.V., L. Yuan, M.S.P. Azevedo, K. Jeong and A.M. Gonzalez et al., 2007. Transfer of maternal cytokines to suckling piglets: In vivo and in vitro models with implications for immunomodulation of neonatal immunity. Vet. Immunol. Immunopathol., 117: 236-248. DOI: 10.1016/j.vetimm.2007.02.013

Razzaque, M.A., T. Al-Mutawa, S. Abbas and M. Bedair, 2009. Performance of pre-weaned dairy calves under hot arid environment: Effects of immunoglobulins and age on diseases and mortality. Am. J. Applied Sci., 6: 1885-1891. DOI: 10.3844/ajassp.2009.1885.1891

Reber, A.J., D.C. Donovan, J. Gabbard, K. Galland and M. Aceves-Avila et al., 2008. Transfer of maternal colostral leukocytes promotes development of the neonatal immune system. I. Effects on monocyte lineage cells. Vet. Immunol. Immunopathol., 123: 186-196. DOI: 10.1016/j.vetimm.2008.01.034

Richards, C.D., 1998. Interleukin-6. In: Mire-Sluis, Thorpe, A.R. (Ed.), Cytokines. Academic Press, San Diego, ISBN: 0-12-498340-5, pp: 87-108.

Scheerlinck, J.P.Y., 1999. Functional and structural comparison of cytokines in different species. Vet. Immunol. Immunopathol., 72: 39-44. DOI: 10.1016/S0165-2427(99)00115-4

Silva, N.A., A.C. Honorio-Franca, F.R. Giachini, L. Mores and E.G.D. Souza et al., 2013. Bioactive factors of colostrum and human milk exhibits a daynight variation. Am. J. Immunol., 9: 68-74. DOI: 10.3844/ajisp.2013.68.74

Souza, F.N.D., E.M.R. Sanchez, M.B. Heinemann, M.A Gidlund and L.D.C. Reis et al., 2012. The innate immunity in bovine mastitis: The role of patternrecognition receptors. Am. J. Immunol., 8: 166-178. DOI: 10.3844/ajisp.2012.166.178

Yamanaka, H., K. Hagiwara, R. Kirisawa and H. Iwai, 2003. Transient detection of proinflammatory cytokines in sera of colostrum-fed newborn calves. J. Vet. Med. Sci., 65: 813-816. PMID: 12939511 